\title{
Curvas antropométricas de recién nacidos chilenos
}

\author{
JAIME ALARCÓN R. ${ }^{1}$, YASNA ALARCÓN V. ${ }^{2}$, ENRIQUE HËRING A. ${ }^{1}$, ROLLY BUCCIONI V. ${ }^{3}$ \\ 1. Médico Pediatra Neonatólogo \\ 2. Médico Cirujano \\ 3. Magíster en Bioestadística \\ Servicio de Neonatología, Hospital Santiago Oriente Luis Tisné Brouse. \\ Servicio de Neonatología, Hospital del Salvador. \\ Servicio de Salud Metropolitano Oriente (SSMO)
}

\begin{abstract}
Anthropometric growth curves in chilean newborns

Objetivo: Design local anthropometric growth curves for selected newborns between 24 and 42 gestational weeks, in order to compare them with other intrauterine growth charts currently used in Chile. Method: 86575 infants born from single pregnancy and with the described gestational age were analyzed. Newborns with maternal or fetal history of delayed intrauterine growth were excluded from de analysis. The average and standard deviation $10^{\text {th }}-50^{\text {th }}-90^{\text {th }}$ percentiles for weight, length and head circumference for each gestational age were calculated, distributing the obtained information in tables and graphics. Comparative statistic analysis with the main perinatal weight/length/head circumference curves currently in use was performed. Results: The obtained anthropometric growth curves of these newborns were compared with Hadlock and Pitalugga charts with statistically significant concordance, whereas there is a statiscally significant difference with Gonzalez and $10^{\text {th }}$ percentile category for premature newborns in the Juez chart. Conclusion: The anthropometric chilean neonatal charts obtained have statistical and clinical advantages in comparison with other newborn local charts currently in use. We recommend these curves for perinatologic clinical use

(Key words: Fetal growth, intrauterine growth curves, birth weight, gestational age, fetal anthropometry). Rev Chil Pediatr 2008; 79 (4): 364-372

\section{RESUMEN}

Objetivo: Diseñar curvas antropométricas locales para recién nacidos seleccionados, entre 24 y 42 semanas de edad gestacional, que permitan un uso clínico confiable, más representativas en comparación con otras curvas de crecimiento intrauterino de uso en Chile. Método: Se analizan 86575 recién nacidos producto de embarazo único, con edad gestacional confiable, excluidos todos aquellos con antecedentes maternos o fetales que afecten el crecimiento intrauterino. Se calculó promedio, desviación estándar, percentiles 10,50 , y 90 para peso, talla y perímetro craneano de cada edad gestacional, tabulando y
\end{abstract}

Trabajo recibido el 02 de mayo de 2007, devuelto para corregir el 19 de junio de 2007, segunda versión el 08 de mayo de 2008, aceptado para publicación el 04 de julio de 2008.

Correspondencia a:

Jaime Alarcón R.

E-mail: jarvadu2004@yahoo.es 
graficando la información. Se realiza análisis comparativo estadístico con las principales curvas de peso perinatal en uso. Resultados: Se obtienen curvas antropométricas de recién nacidos chilenos entre 24 y 42 semanas de población seleccionada. La comparación estadística de los pesos revela semejanza con las curvas de Hadlock y Pittaluga, y diferencias significativas con las curvas de González y percentil 10 de los recién nacidos de pretérmino de la curva de Juez. Conclusión: Se obtienen curvas antropométricas chilenas, con ventajas comparativas con respecto a otras curvas locales neonatales. Se recomienda su uso clínico perinatológico.

(Palabras clave: crecimiento fetal, curvas crecimiento intrauterino, peso al nacer, edad gestacional, antropometría fetal).

Rev Chil Pediatr 2008; 79 (4): 364-372

\section{Introducción}

En 1963, Lubchenco y cols ${ }^{1}$, confeccionaron una curva de crecimiento intrauterino (CIU) a partir de recién nacidos $(\mathrm{RN})$ norteamericanos entre 24 y 42 semanas de edad gestacional. Esta curva se ha utilizado extensamente como parámetro de referencia tanto para la población norteamericana como para otras poblaciones, incluida la chilena.

Posteriormente, en 1967, Battaglia-Lubchenco², clasificaron a los $\mathrm{RN}$ según la relación entre su edad gestacional y peso de nacimiento, como grande, adecuado o pequeño para su edad gestacional, mismos parámetros que se utilizan actualmente para identificar a los grupos de mayor riesgo perinatológico en cuanto a morbimortalidad.

La OMS $1970^{3}$ recomienda el diseño de curvas de CIU en cada uno de los centros perinatológicos, con el objeto que representen las características particulares de cada población.

La Sociedad Chilena de Pediatría publica en $1983^{4}$, las recomendaciones para la evaluación del CIU en RN chilenos, proponiendo los criterios que debían incorporar estos estudios para obtener una curva representativa de nuestra realidad nacional. Estos criterios incluyen un número mínimo de 100 neonatos por cada edad de gestación, edad gestacional confiable (obstétrica y pediátrica) y la exclusión de factores que afecten el CIU de manera conocida y significativa.

En Chile, varios autores han publicado curvas neonatales, poblacionales o ecográficas, cada una con beneficios y limitaciones en su uso clínico ${ }^{5-14}$ La curva publicada por Juez en 1984 ha sido utilizada masivamente en los últimos años. Cabe destacar que a pesar de ser una curva neonatal en base a una población seleccionada, la población reclutada en edades gestacionales menores a 36 semanas, donde el riesgo de morbimortalidad perinatal es mayor, resulta insuficiente para permitir conclusiones absolutas, lo cual pone en discusión su uso clínico para edades gestacionales extremas ${ }^{13}$.

Por otra parte, la curva publicada por González el año 2004, si bien cumple con los criterios definidos por la OMS, se trata de un análisis realizado en base a datos del Instituto Nacional de Estadísticas, sin exclusión de RN con patologías maternas o fetales que afecten el desarrollo normal del RN, lo cual implica un riesgo de subdiagnóstico de RN PEG en su uso clínico, tema que ya ha sido planteado e investigado por otros autores, reafirmando esta hipótesis ${ }^{14-16}$.

En la actualidad, considerando la sobrevida que se ha logrado en edades gestacionales extremas $^{17-19}$, es importante poder contar con tablas antropométricas confiables, que contemplen un mayor número de casos, evitando el sesgo que se pudiera producir al continuar aplicando los parámetros publicados hasta ahora.

Desde 1988, el servicio de Neonatología del Hospital del Salvador, trasladado posteriormente al Hospital Luis Tisné, registra en una base de datos, la información de todos los partos ocurridos en la maternidad del establecimiento. La gran casuística recopilada en este centro de alta complejidad, donde el número de partos es considerable, y por ende, el de RN de EG extremas sigue el mismo patrón, motiva a crear 
curvas antropométricas en población seleccionada para edades gestacionales entre 24 y 42 semanas.

Considerando las ventajas comparativas en cuanto al número de $\mathrm{RN}$ para cada edad gestacional y la selección acuciosa de los pacientes ingresados en el estudio, se espera obtener curvas de antropometría neonatal que representen mejor a $\mathrm{RN}$ chilenos sin patología asociada, en comparación con otras curvas de uso perinatológico.

\section{Material y Método}

Se analiza una población de 148395 recién nacidos del Servicio de Salud Metropolitano Oriente (SSMO), de las maternidades del Hospital del Salvador entre los años 1988-2000 y Hospital Luis Tisné entre los años 2001-2005.

Se recogió la información en base de datos File Maker Pro 3.0 v3, creando matrices en planillas Excel, donde fue analizada la información.

Se registraron antecedentes obstétricos: Fecha última regla (FUR), morbilidad materna asociada al embarazo, y datos neonatales: peso, talla, perímetro cefálico, EG pediátrica, sexo, gemelaridad y patología perinatal.

En la atención inmediata del RN, se realiza antropometría neonatal estandarizada, por matronas calificadas y evaluación pediátrica de EG según impresión clínica de pediatras o neonatólogos de experiencia ${ }^{4}$. En RN menores de 32 semanas se les realizó reevaluación de EG antes de las $72 \mathrm{~h}$ de vida, por norma de atención.

Fueron excluidos del estudio todos aquellos RN hijos de madre con patología asociada como Diabetes Mellitus, Diabetes Gestacional, Síndrome Hipertensivo del Embarazo, Colestasia Intrahepática, Infección Ovular, RH negativo sensibilizadas, embarazos múltiples, deterioro de la unidad feto-placentaria y malformaciones congénitas mayores. Se omitieron los casos con información incompleta, errores de digitación o sin edad gestacional confiable.

Se consideró EG confiable, a la EG por FUR coincidente en un margen de no más de 10 días con la EG pediátrica ${ }^{2}$, validándose la
FUR. Las semanas de EG confiable se consignan al número entero inferior.

Se clasificó la población según EG y sexo en tablas de Peso, Talla, Perímetro Cefálico, e Índice Ponderal (IP). Se calculó promedios, desviación estándar y distribución por percentiles 10,50 y 90 . Se ajustó la información a polinomios de 3er orden y se evaluó la significación estadística por $\mathrm{T}$ de student a la diferencia de los promedios del peso por sexo, respecto al promedio general. Se consideró significativo $p<0,05$.

Se comparan nuestros resultados con la información publicada disponible de las tablas de González, Juez, Pittaluga y Hadlock H,7,13,14,20. $^{2}$ Los promedios de cada curva son comparados mediante la prueba T de Student. Se comparan las DS calculando la razón de varianza entre ellas y se analiza la distribución $\mathrm{F}$ para evaluar la significación estadística de la diferencia entre las DS de las distintas curvas.

Con el fin de objetivar la importancia clínica de la diferencia entre las dispersiones de las curvas, se realiza un análisis comparativo aplicando la Prueba $\mathrm{T}$ pareada entre los percentiles 10 de las distintas curvas.

\section{Resultados}

De un total de $148395 \mathrm{RN}$ registrados, 86575 (58,34\%) cumplieron con los criterios de selección para el análisis de peso, talla e índice ponderal, de los cuales el 51,1\% corresponden a sexo masculino.

El detalle de los resultados obtenidos tras el análisis de peso y talla, se muestra en la tabla 1 , junto con el IP resultante de la relación pesotalla. Se tabula el número de RN para cada EG, promedio, DS de pesos y tallas, y los percentiles 10,50 y 90 , ajustados a polinomio de tercer orden, donde hallamos una concordancia entre datos reales y alisados mayor al $99 \%\left(\mathrm{R}^{2}>0,99\right)$.

En la figura 1 se muestra la curva de peso de acuerdo a EG y los percentiles 10, 25, 50, 75 y 90 ; y en el recuadro, la diferencia de los promedios por sexo respecto a la media común entre las 35 y 42 semanas, tramo en que la diferencia es significativa (Prueba T de Student).

Para perímetro cefálico, 82604 (55,66\%) $\mathrm{RN}$ fueron seleccionados, excluyéndose un 
Tabla 1. Promedios, Desviación Estándar y Percentiles del Peso, Talla e Indice Ponderal, RN seleccionados. Significación estadística para la diferencia de los pesos por Sexo

\begin{tabular}{|c|c|c|c|c|c|c|c|c|c|c|c|c|c|c|}
\hline \multirow{2}{*}{$\begin{array}{l}\text { Edad } \\
\text { gesta- } \\
\text { cional }\end{array}$} & \multirow[t]{2}{*}{$\mathbf{n}$} & \multirow{2}{*}{$\begin{array}{c}\text { Peso } \bar{x} \\
\text { ambos } \\
\text { sexos }\end{array}$} & \multirow{2}{*}{ DS } & \multirow{2}{*}{$\begin{array}{c}\text { Sexo } \\
t \\
\text { Student }\end{array}$} & \multirow{2}{*}{ p10 } & \multirow[t]{2}{*}{ p50 } & \multirow[t]{2}{*}{ p90 } & \multirow{2}{*}{$\begin{array}{c}\text { Talla } \\
\bar{x} \\
\text { general }\end{array}$} & \multirow[t]{2}{*}{ DS } & \multirow[t]{2}{*}{ p10 } & \multirow[t]{2}{*}{ p50 } & \multirow[t]{2}{*}{ p9o } & \multicolumn{2}{|c|}{$\begin{array}{l}\text { Indice } \\
\text { Ponderal }\end{array}$} \\
\hline & & & & & & & & & & & & & p10 & p90 \\
\hline 24 & 41 & 765,4 & 105,1 & NS & 630,0 & 749,9 & 898,6 & 31,7 & 2,2 & 30,0 & 31,4 & 34,1 & 1,869 & 2,539 \\
\hline 25 & 39 & 817,7 & 118,9 & NS & 661,2 & 809,6 & 965,6 & 33,1 & 2,2 & 31,1 & 32,9 & 35,7 & 1,905 & 2,568 \\
\hline 26 & 58 & 907,7 & 135,7 & NS & 727,8 & 904,9 & 1074,1 & 34,6 & 2,2 & 32,3 & 34,5 & 37,2 & 1,941 & 2,598 \\
\hline 27 & 66 & 1030,8 & 155,0 & NS & 825,8 & 1031,6 & 1219,0 & 36,1 & 2,2 & 33,6 & 36,0 & 38,7 & 1,977 & 2,627 \\
\hline 28 & 84 & 1182,3 & 176,3 & NS & 950,8 & 1185,2 & 1395,1 & 37,6 & 2,2 & 35,0 & 37,5 & 40,1 & 2,013 & 2,657 \\
\hline 29 & 80 & 1357,6 & 199,1 & NS & 1098,6 & 1361,3 & 1597,2 & 39,0 & 2,1 & 36,3 & 39,0 & 41,6 & 2,048 & 2,686 \\
\hline 30 & 86 & 1552,2 & 223,0 & NS & 1265,0 & 1555,4 & 1820,1 & 40,5 & 2,1 & 37,7 & 40,5 & 43,0 & 2,084 & 2,715 \\
\hline 31 & 126 & 1761,3 & 247,4 & NS & 1445,8 & 1763,2 & 2058,8 & 41,9 & 2,0 & 39,1 & 41,9 & 44,3 & 2,120 & 2,745 \\
\hline 32 & 190 & 1980,3 & 272,0 & NS & 1636,7 & 1980,1 & 2308,0 & 43,2 & 1,9 & 40,5 & 43,3 & 45,6 & 2,156 & 2,774 \\
\hline 33 & 265 & 2204,5 & 296,1 & NS & 1833,6 & 2201,9 & 2562,6 & 44,5 & 1,9 & 41,8 & 44,6 & 46,8 & 2,192 & 2,804 \\
\hline 34 & 466 & 2429,5 & 319,3 & NS & 2032,2 & 2424,1 & 2817,5 & 45,7 & 1,8 & 43,1 & 45,8 & 47,9 & 2,228 & 2,833 \\
\hline 35 & 892 & 2650,4 & 341,2 & $\left({ }^{*}\right)$ & 2228,3 & 2642,2 & 3067,3 & 46,8 & 1,7 & 44,2 & 46,9 & 49,0 & 2,264 & 2,862 \\
\hline 36 & 2230 & 2862,8 & 361,2 & $\left({ }^{*}\right)$ & 2417,7 & 2851,8 & 3307,1 & 47,9 & 1,7 & 45,3 & 48,0 & 49,9 & 2,300 & 2,892 \\
\hline 37 & 6482 & 3061,9 & 378,9 & $\left({ }^{*}\right)$ & 2596,2 & 3048,5 & 3531,6 & 48,8 & 1,6 & 46,3 & 48,9 & 50,8 & 2,336 & 2,921 \\
\hline 38 & 17243 & 3243,1 & 393,8 & $(*)$ & 2759,5 & 3228,0 & 3735,7 & 49,6 & 1,5 & 47,2 & 49,6 & 51,5 & 2,372 & 2,951 \\
\hline 39 & 25793 & 3401,8 & 405,4 & $\left({ }^{*}\right)$ & 2903,4 & 3385,7 & 3914,1 & 50,3 & 1,5 & 47,9 & 50,3 & 52,1 & 2,407 & 2,980 \\
\hline 40 & 21562 & 3533,3 & 413,2 & $\left({ }^{*}\right)$ & 3023,7 & 3517,2 & 4061,8 & 50,9 & 1,4 & 48,5 & 50,8 & 52,6 & 2,443 & 3,009 \\
\hline 41 & 9956 & 3633,1 & 416,8 & $\left({ }^{*}\right)$ & 3116,2 & 3618,2 & 4173,6 & 51,3 & 1,4 & 48,8 & 51,1 & 52,9 & 2,479 & 3,039 \\
\hline 42 & 916 & 3696,5 & 415,6 & $\left({ }^{*}\right)$ & 3176,7 & 3684,2 & 4244,3 & 51,6 & 1,3 & 49,0 & 51,2 & 53,0 & 2,515 & 3,068 \\
\hline Total & 86.575 & & & & & & & & & & & & & \\
\hline
\end{tabular}

Distribución de la población en estudio, promedio general de peso y talla para cada EG y su DS; percentiles 10,50, y 90 ajustados a polinomios de tercer orden $(R 2=0,99)$, percentiles 10 y 90 del Índice Ponderal ajustados a su tendencia lineal $(R 2=$ $0,94)$ y significación estadística de la prueba t de Student para las diferencias de los promedios respecto a la media común de los sexos. $\left({ }^{*}\right) p<0,05$.

mayor número fundamentalmente debido a la falta de registro. Se detalla en la tabla 2 el número de RN, promedio, DS y percentiles 10, 50 y 90 ajustados a polinomio de tercer orden $\left(\mathrm{R}^{2}>0,99\right)$ para cada $E G$, obteniendose una curva que se ve graficada en la figura 2 , en conjunto con la curva de tallas para cada edad gestacional.

La tabla 3 compara los promedios y DS de la curva de peso obtenida, con respecto a las curvas de uso frecuente. Los promedios no resultan significativamente distintos con las curvas de Juez y Pittaluga, pero sí con la curva poblacional de Gonzalez entre las 31 a 39 semanas, donde los promedios son significativamente inferiores (Prueba $\mathrm{T}$ de Student), lo que repercute en desplazar la 2 da DS y percentiles inferiores, que son los valores que se utilizan como referencia de las poblaciones de riesgo por bajo peso neonatal.

Como medida de dispersión, utilizamos el porcentaje que implica la DS con respecto al promedio (Coeficiente de Variación). En nues-
Tabla 2. Perimetro Cefálico (PC): Promedio, DS y Percentiles; RN seleccionados

\begin{tabular}{|c|c|c|c|c|c|c|}
\hline \multirow{2}{*}{$\begin{array}{c}\text { Edad } \\
\text { Gestaciona }\end{array}$} & \multirow[b]{2}{*}{$\mathbf{n}$} & \multirow[b]{2}{*}{$\bar{x}$} & \multirow[b]{2}{*}{ DS } & \multicolumn{3}{|c|}{ PC Percentiles } \\
\hline & & & & p10 & p50 & p9o \\
\hline 24 & 6 & 23,0 & 1,0 & 21,9 & 23,2 & 24,4 \\
\hline 25 & 13 & 24,1 & 1,2 & 22,8 & 24,3 & 25,7 \\
\hline 26 & 13 & 25,1 & 1,3 & 23,6 & 25,3 & 26,9 \\
\hline 27 & 16 & 26,2 & 1,4 & 24,5 & 26,4 & 28,1 \\
\hline 28 & 26 & 27,2 & 1,5 & 25,5 & 27,4 & 29,1 \\
\hline 29 & 23 & 28,1 & 1,5 & 26,4 & 28,3 & 30,1 \\
\hline 30 & 37 & 29,1 & 1,5 & 27,3 & 29,3 & 31,0 \\
\hline 31 & 68 & 30,0 & 1,5 & 28,1 & 30,2 & 31,8 \\
\hline 32 & 143 & 30,8 & 1,5 & 29,0 & 31,0 & 32,6 \\
\hline 33 & 226 & 31,6 & 1,4 & 29,8 & 31,8 & 33,3 \\
\hline 34 & 412 & 32,3 & 1,4 & 30,5 & 32,5 & 33,9 \\
\hline 35 & 799 & 33,0 & 1,3 & 31,2 & 33,1 & 34,5 \\
\hline 36 & 2128 & 33,6 & 1,3 & 31,9 & 33,7 & 35,0 \\
\hline 37 & 6193 & 34,1 & 1,2 & 32,4 & 34,2 & 35,5 \\
\hline 38 & 16458 & 34,5 & 1,2 & 32,9 & 34,6 & 35,9 \\
\hline 39 & 24752 & 34,9 & 1,2 & 33,2 & 34,9 & 36,2 \\
\hline 40 & 20760 & 35,1 & 1,3 & 33,4 & 35,1 & 36,5 \\
\hline 41 & 9625 & 35,2 & 1,4 & 33,6 & 35,2 & 36,8 \\
\hline \multirow[t]{2}{*}{42} & 906 & 35,3 & 1,5 & 33,5 & 35,2 & 37,0 \\
\hline & 82604 & & & & & \\
\hline
\end{tabular}

Distribución de la población en estudio, promedio general de perímetros cefálicos para cada EG y su DS; percentiles 10,50 y 90 ajustados a un polinomio de tercer orden $(R 2=0,98)$ 


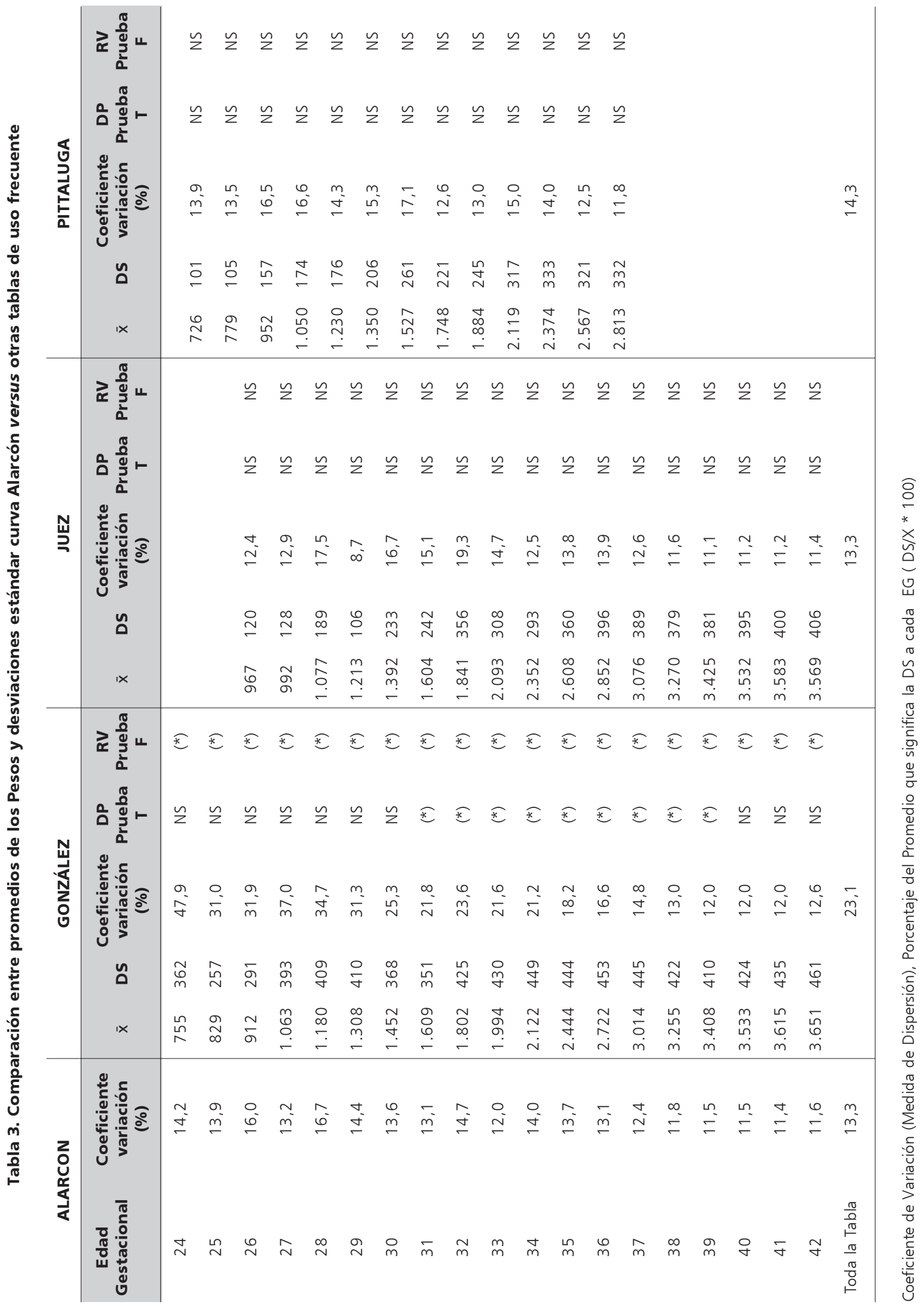


tra curva, como en las otras curvas de población seleccionada, la dispersión oscila entre el $9 \%$ y el $17 \%$, sin embargo, en la curva de González, bajo las 34 semanas, resulta superior al $21 \%$ llegando a un máximo de $47,9 \%$ a las 24 semanas, lo que le resta confiabilidad estadística.

La Razón de Varianza de las DS, no demuestra diferencia significativa con las otras curvas de población seleccionada neonatales y ecográfica, lo que las hace similares en su distribución respecto a los promedios y DS, pero sí muestra diferencia significativa para toda la curva de González, lo que la calificaría como una curva diferente estadísticamente.

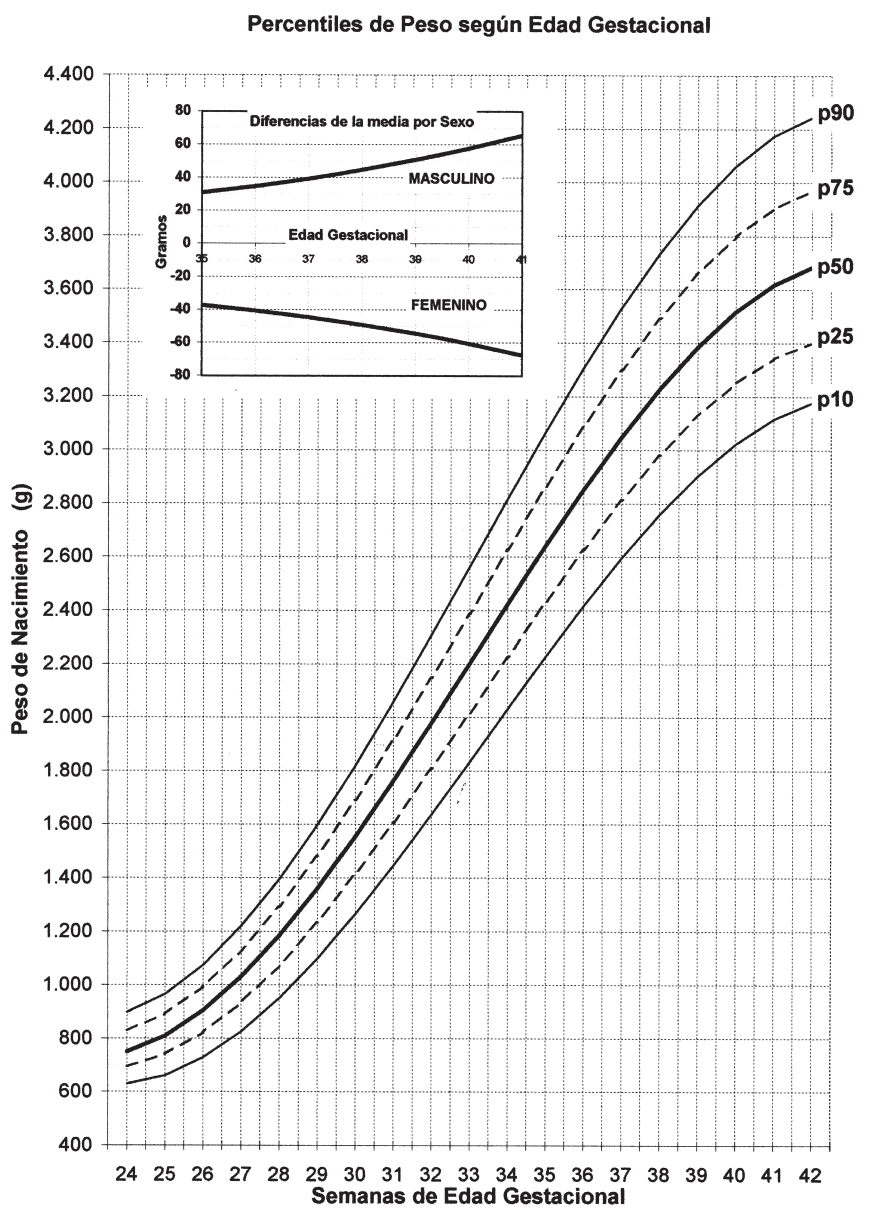

Figura 1. $86575 \mathrm{RN}$ de ambos sexos, población seleccionada, Edad Gestacional (EG) confiable por evaluación pediátrica y FUR coincidente.
El análisis comparativo del p10 que se muestra en la tabla 4, demuestra una diferencia significativa en el rango de $\mathrm{RN}$ de término con respecto a la curva de González, y en el rango de RN de pretérmino, con las curvas de Juez y González, lo que se traducirá en un subdiagnóstico de $\mathrm{RN}$ pequeño para la edad gestacional (PEG). Se grafica en la figura 3 el p10 en las EG de pretérmino, para obtener una imagen visual de las diferencias.

\section{Discusión}

Las curvas antropométricas presentadas, al ser obtenidas con un número importante de casos seleccionados, y con una coincidencia de alisamiento estadístico cercano al 99\% de la data cruda, representa una información real y actualizada de la antropometría de RN chilenos.

En cuanto a sexos, resulta práctica la utilización de una curva única, ya que las diferencias son significativas solo después de las 35 semanas de EG, lo cual puede corregirse con la información adjunta en el recuadro de la figura 1.

En relación a la corrección por otras variables biológicas como son la talla materna y la paridad, estas no fueron consideradas en nuestro trabajo por información insuficiente, pero podrían utilizarse los datos previamente publicados ${ }^{21,22}$.

Con respecto al análisis comparativo con la curva de peso de González, si bien cumple con los requisitos sugeridos por la OMS en cuanto en número de $\mathrm{RN}$ para cada EG, al ser un estudio poblacional, no excluye a RN con patología asociada que afecten el CIU, lo que se traduce en pesos promedios inferiores y una gran dispersión de la información y por lo tanto, un riesgo de subdiagnóstico de RN PEG en su aplicación clínica. 
Tabla 4. Significación estadística de las diferencias del percentil 10 del Peso de Nacimiento por rangos de edad gestacional entre tabla Alarcón versus tablas de uso frecuente

\begin{tabular}{|c|c|c|c|c|}
\hline $\begin{array}{l}\text { Rangos } \\
\text { de edad } \\
\text { gestacional }\end{array}$ & González & Juez & Pittaluga & Hadlock \\
\hline $\begin{array}{l}\text { Rango } 24-35 \\
\text { semanas }\end{array}$ & $p<0,05$ & $p<0,05$ & NS & NS \\
\hline $\begin{array}{l}\text { Rango } 36-42 \\
\text { semanas }\end{array}$ & $p<0,05$ & NS & Sin datos & NS \\
\hline Toda la Curva & $p<0,05$ & $p<0,05$ & NS & NS \\
\hline
\end{tabular}

Prueba T pareada para las diferencias del percentil 10 en dos rangos de EG.

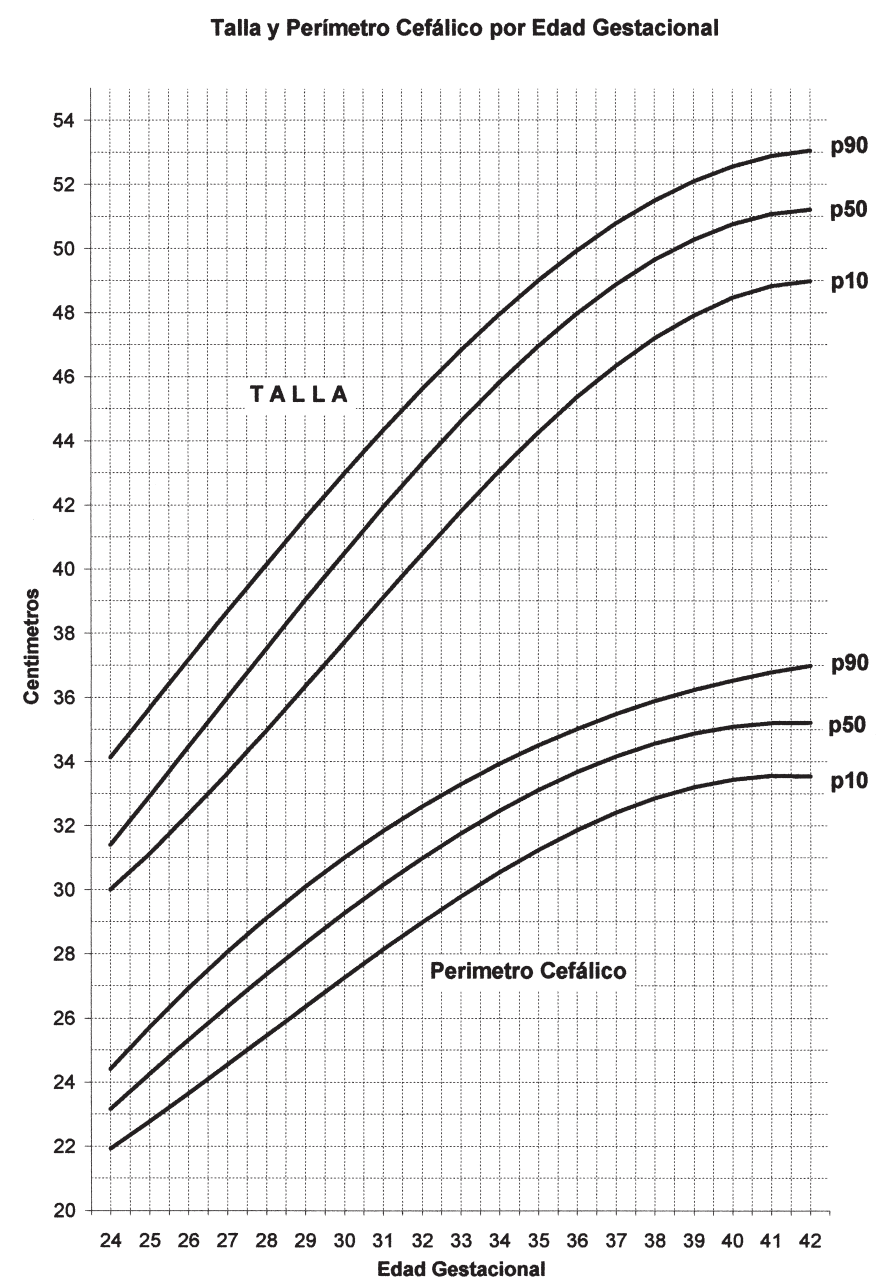

Figura 2. Recién nacidos seleccionados, de ambos sexos, edad gestacional confiable.
Publicaciones recientes han demostrado que su uso implica una disminución de hasta un $50 \%$ del diagnóstico de RN PEG ${ }^{23}$, con respecto a la curva de Juez y a la vez, aumento de las hospitalizaciones y morbimortalidad neonatal asocia$\mathrm{da}^{15}$.

Algo similar se ha observado en un estudio de peso estimado por ecografía, donde fetos evaluados como bajo el p10 por curvas ecográficas, resultan normales por la curva poblacional de González. El seguimiento ecográfico mostró severas alteraciones de la circulación feto-placentaria de algunos de estos casos, poniendo de manifiesto un riesgo de subdiagnóstico de restricción de crecimiento, debido a la baja exigencia de la curva poblacional ${ }^{16}$.

Considerando que es en los RN de pretérmino donde se concentra el mayor riesgo de morbimortalidad, realizamos un análisis específico para este rango de EG, donde demostramos una diferencia significativa con el p10 de la curva de Juez, probablemente relacionada al escaso número de $\mathrm{RN}$ prematuros reclutados para dicha curva.

En las curvas del presente estudio, si bien el número de RN aun no alcanza los 200 para cada EG sugeridos por las OMS para considerar una curva como estándar, la absoluta coincidencia con el estudio de Pittaluga sugieren la posibilidad de sumar ambos resultados y validar una curva única de referencia.

En cuanto a la curva ecográfica de Hadlock, la comparación con nuestra curva no revela diferencias significativas en cuanto a distribución de percentiles, lo que las hace similares en el uso clínico permitiendo la continuidad de la evaluación perinatal. 
Percentil 10 del Peso Recién Nacidos Pretérmino multiples curvas

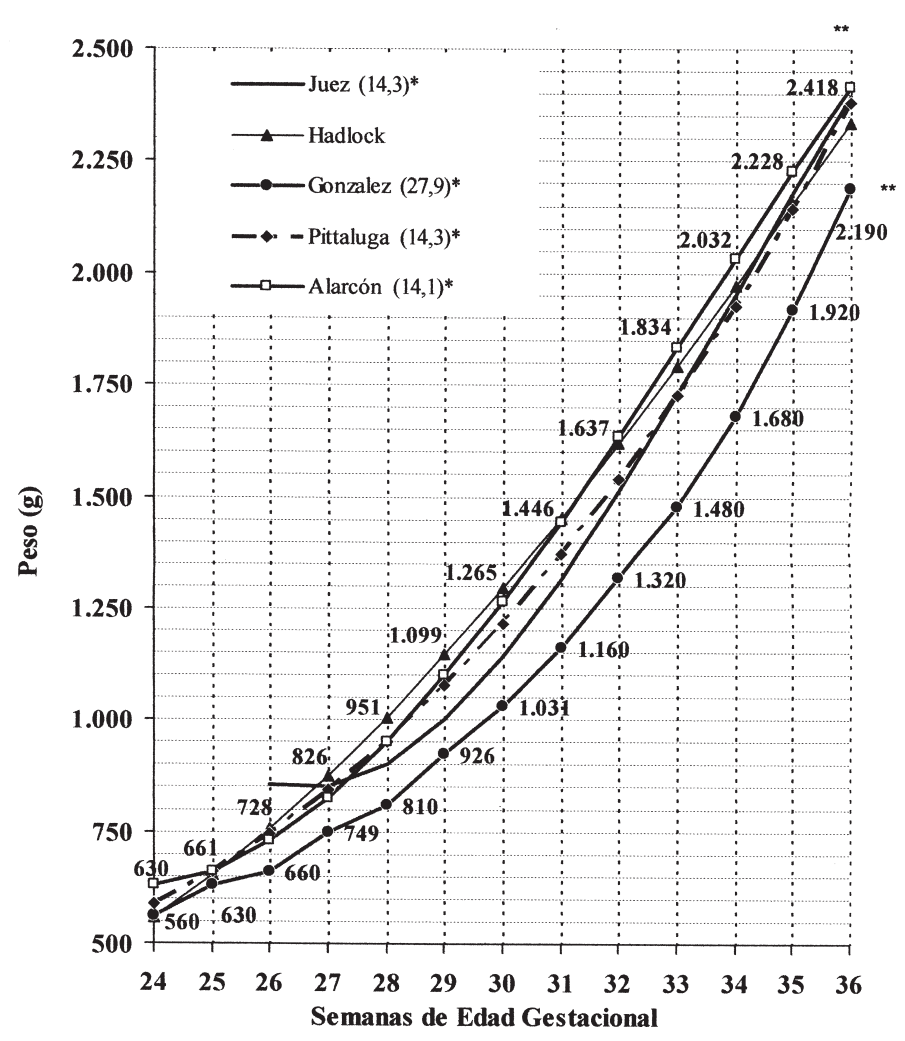

Figura 3. * Dispersión promedio de cada curva, hasta las 36 semanas de EG. Gráfico que muestra el Percentil 10 de las curvas de Crecimiento Intrauterino en Recién Nacidos de pretérmino, compara la curva Alarcón con curvas de Hadlock, Pittaluga, Juez y González, se detalla el valor numérico del peso para cada Edad Gestacional de las Curvas Alarcón y González.

\section{Conclusiones}

El elevado número de casos y la cuidadosa selección de la población analizada, han permitido obtener curvas antropométricas representativas de la población chilena, con ventajas comparativas con respecto a otras curvas de peso neonatal de uso habitual, lo que las hace confiables para su uso clínico perinatológico. Sugerimos el uso clínico de estas curvas como estándar referencial.

\section{Referencias}

1.- Lubchenco LO, Hansman C, Dressler M, Boyd E: Intrauterine growth as estimated from liveborn birthweight data at 24 to 42 weeks of gestation. Pediatrics
1963; 32: 793-800.

2.- Battaglia F, Lubchenco L: A practical clasification of newborn infants by weight and gestational age. J Pediatr 1967; 71: 129-33.

3.- OMS: Prevención de la mortalidad y morbilidad perinatales. Informe de un Comité de Expertos de la OMS. Serie de Informes Técnicos No 457, 1970.

4.- Alvear J, Beca JP, Saavedra G, et al: Evaluación del crecimiento intrauterino del recién nacido. Rev Chil Pediatr 1983: 54: 374-8.

5.- Rizzardini M, Silva E, Schildlow D: Curva de crecimiento intrauterino en recién nacidos chilenos. Rev Chil Pediatr 1972; 43: 7-13.

6.- Juez G, Ventura-Juncá P, Lucero E: Crecimiento intrauterino en un grupo seleccionado de recién nacidos chilenos. Rev Méd Chile 1984; 112: 759-64.

7.- Juez G, Lucero E, Ventura-Juncá P: Crecimiento intrauterino en recién nacidos chilenos de clase media. Rev Chil Pediatr 1989; 60 (4): 198-202.

8.- Beca JP, Rizzardini M, Weldt E, Corvalán S: Talla peso y perímetro craneano según edad gestacional en 
recién nacidos menores de 35 semanas. Rev Chil Pediatr 1989; 60: 1-5

9.- Juez G, Lucero E, Ventura-Juncá P, Galleguillos J: Talla, Circunferencia Craneana e Indice Ponderal en Recién Nacidos Chilenos de Clase Media. Rev Chil Pediatr 1993; 64 (4): 237-40.

10.- Vaccaro CH: Crecimiento Fetal. Rev Chil Ostet Ginecol 1991; 56 (5): 353-8

11.- Lagos R, Espinoza R, Echeverría P, Orellana J: Gráfica Regional de Crecimiento Fetal. Comparación con estándar internacional. Rev Chil Obstet Ginecol 1998; 63 (5): 370-3.

12.- Krausse S, Grob J, Campos G: Características Antropométricas de recién nacidos vivos en Valdivia en comparación a patrón de Battaglia y Lubchenco. Rev Latin Perinat 1984; 4: 111-8

13.- Pittaluga PE, Díaz AV, Mena NP, et al: Curva de crecimiento intrauterino para prematuros entre 23 a 36 semanas de edad gestacional. Rev Chil Pediatr 2002; 73 (2): 135-41. ISSN 0370-4106.

14.- González P, Rogelio, Gómez M, Ricardo, Castro S, René, et al: Curva nacional de distribución de peso al nacer según edad gestacional: Chile, 1993 a 2000. Rev Méd Chile 2004; 132 (10): 1155-65. ISSN 00349887.

15.- Carvajal JA, Morales Y, Germain A: Restricción del Crecimiento Fetal: Un hallazgo frecuente entre los RN prematuros. Rev Chil Obstet Ginecol 2001; 66: 463-71.

16.- Leyton M, Gana J, Bardi E: Evaluación de Crecimiento Fetal. Rev de Obstet Ginecol Hosp Stgo Oriente Dr Luis Tisné 2007; 2 (1): 2-16.

17.- González R, Merialdi $M$, Lincetto $O$, et al: Reduction in Neonatal Mortality in Chile Between 1990 and 2000. Pediatrics 2006; 117: e949-e954

18.- Lawn JE, Cousens S, Zupan J: Lancet Neonatal Survival Steering Team. 4 million neonatal deaths: When? Where? Why? Lancet 2005; 365: 891-900.

19.- Morgues M, Henríquez MT, Toha D, et al: Sobrevida del niño menor de 1500 g en Chile. Rev Chil Obstet Ginecol 2002; 67: 100-5.

20.- Hadlock FP, Harris RB, Martínez-Poyer J: In útero análisis of fetal growth: A sonographic weight standard. Radiology 1991; 181: 129-33.

21.- Juez G, Lucero E, Ventura-Juncá P: Crecimiento In- trauterino según sexo fetal y paridad materna. Rev Chil Pediatr 1989; 60: 198-202.

22.- Juez G, Opazo A, Lucero E: Influencia de la talla materna sobre el crecimiento fetal. Rev Chil Obstet Ginecol 1990; 55: 104-8

23.- Lagos R, et al: Subdiagnóstico del RN PEG. Rev Hosp Infantil Ramón Sardá 2004; 23 (1).

24.- Lubchenco LO, Hansman C, Boyd E: Intrauterine growth in length and head circunference as estimated from livebirths as gestacional ages from 26 to 42 weeks. Pediatrics 1966; 37: 403-10.

25.- Hadlock FP, Harrist RB, Sharman RS, et al: Estimation of fetal weight with the use of head, body, and femur measurements - a prospective study. Am J Obstet Gynecol 1985; 151 (3): 333-7

26.- Dudley N. A systematic review of the ultrasound estimation of fetal weight. Ultrasound Obstet Gynecol 2005; 25: 80-89.

27.- OMS. Physical Status: The Use and Interpretation of Anthropometry. Report of a WHO Expert Committee. Technical Report Series, No 854. 1995

28.- Battaglia FC, Frazier TM, Hellegers AE: Birth weight, gestational age and pregnancy outcome, with special reference to high birth weight-low gestational age Infant. Pediatrics 1966 37: 417-22.

29.- Williams RL, Creasy RK, Cunningham GC, Hawes WE, Norris FD, Tashiro $M$ : Fetal growth and perinatal viability in California. Obstet Gynecol 1982 59: 624-32.

30.- Bukowski R, Gahn D, Denning J, Saade G: Impairment of growth in fetuses destined to deliver preterm. Am J Obstet Gynecol 2001; 185 (2): 463-7.

31.- Kramer M, Olivier M, Mc Lean F, Dougherty GE, Willis D, Usher R: Determinants of fetal growth and body proportionality. Pediatrics 1990, 86 (1): 18-26.

32.- Arnold CC, Kramer MS, Hobbs CA, McLean FH, Usher $R H$ : Very Low Birth Weight: A Problematic Cohort for Epidemiologic Studies of Very Small or Immature Neonates. Am J Epidemiol 1991; 134: 604-13.

33.- Brenner WE, Edelman D, Hendrics C: A standard of fetal growth for the United States of America. Am J Obstet Gynecol 1976; 126: 555-64.

34.- Mühlhausen $M G$, Navarrete GC: Malnutrición intrauterina: comparación de curvas de crecimiento en la detección de alto riesgo neonatal. Rev Chil Pediatr 1993; 64 (1): 26-30. ISSN 0370-4106. 